\title{
Gaussian binomial coefficients
}

\author{
A. G. Shannon \\ Warrane College \\ The University of New South Wales \\ Kensington, NSW 2033, Australia \\ e-mails: t. shannonewarrane.unsw.edu.au, \\ tshannon38@gmail.com
}

Received: 11 December 2019

Accepted: 4 February 2020

\begin{abstract}
This paper extends Gaussian binomial coefficients (and so-called) Fibonomial coefficients) with identities related to Horadam's generalized binomial coefficients.

Keywords: Gaussian binomial coefficients, Generalized Fibonacci numbers, Fibonomial coefficients.
\end{abstract}

2010 Mathematics Subject Classification: 11B65, $11 B 39$.

\section{Introduction}

Gaussian binomial coefficients and associated $q$-series have been treated in many papers in a variety of applications by Carlitz $[5,6,7,8,10]$ which display some typical settings for the series, so is there anything more that can be said? These Gaussian numbers are different from, but have some analogous properties to, 'Gaussian Integers' properly so-called [11].

$q$-series are defined basically by

$$
(q)_{n}=(1-q)\left(1-q^{2}\right) \ldots\left(1-q^{n}\right), n>0,(q)_{0}-1 .
$$

Arising out of these are Gaussian binomial coefficients

$$
\begin{gathered}
\left(\begin{array}{l}
n \\
k
\end{array}\right)_{q}=\left\{\begin{array}{cc}
\frac{\left(1-q^{n}\right)\left(1-q^{n-1}\right) \ldots\left(1-q^{n-k+1}\right)}{(1-q)\left(1-q^{2}\right) \ldots\left(1-q^{k}\right)}, & k \leq n, \\
0, & k>n,
\end{array}\right. \\
=\frac{[n]_{q} !}{[k]_{q} ![n-k]_{q} !},(k \leq n)
\end{gathered}
$$

where 


$$
\begin{aligned}
{[n]_{q} } & =1+q+q^{2}+\cdots+q^{n-1} \\
& =\left\{\begin{array}{cl}
\frac{1-q^{n}}{1-q}, & q \neq 1, \\
n, & q=1 .
\end{array}\right.
\end{aligned}
$$

Mercier [18] has already developed some properties in his theorem and its corollaries. It is proposed here to develop some new results with generalized Fibonacci numbers $\left\{U_{n}\right\} \equiv\left\{U_{n}(a, b ; p, q\}[16]\right.$ where $p, q$ are arbitrary integers, and $a, b$ are initial values.

\section{Fibonomial coefficients}

Carlitz [9] and Horadam [16] have used them in the form that follows with generating functions for powers of generalized Fibonacci numbers. If we formally let $q=\beta / \alpha$ in the above definition, where $\alpha, \beta$, assumed distinct, are the roots of $x^{2}-p x+q=0$, then

$$
\begin{gathered}
\left(\begin{array}{l}
n \\
k
\end{array}\right)_{q}=\frac{\left(1-(\beta / \alpha)^{n}\right) \ldots\left(1-(\beta / \alpha)^{n-k+1}\right)}{\left(1-(\beta / \alpha)^{1}\right)\left(1-(\beta / \alpha)^{2}\right) \ldots\left(1-(\beta / \alpha)^{k}\right)} \\
=\alpha^{k(n-k)} \frac{U_{n} U_{n-1} \ldots U_{n-k+1}}{U_{1} U_{2} \ldots U_{k}} \\
=U_{n} C_{n, k} \alpha^{k(n-k)},
\end{gathered}
$$

in which the sequence $\left\{U_{n}\right\}$ defined above, and

$$
C_{n, k}=\frac{U_{n-1} \ldots U_{n-k+1}}{U_{1} U_{2} \ldots U_{k}} .
$$

The significance of the $C_{n, k}$ can be seen in Hoggatt $[13,14]$ in which he developed properties for ordinary Fibonacci numbers and the Gaussian binomial coefficients, there called Fibonomial coefficients [12, 17]. Some of these properties were prefigured by Alexanderson [1] and Andrews [4].

In this spirit we obtain

\section{Theorem 1.}

Proof:

$$
\left(\begin{array}{c}
n-1 \\
k
\end{array}\right)_{q}+\left(\begin{array}{l}
n-1 \\
k-1
\end{array}\right)_{q}=\frac{2-q^{k}-q^{n-k}}{1-q^{n}}\left(\begin{array}{l}
n \\
k
\end{array}\right)_{q} .
$$

$$
\begin{aligned}
\left(\begin{array}{c}
n-1 \\
k
\end{array}\right)_{q} & +\left(\begin{array}{l}
n-1 \\
k-1
\end{array}\right)_{q}=\frac{\left(1-q^{n-1}\right)\left(1-q^{n-2}\right) \ldots\left(1-q^{n-k}\right)}{(1-q)\left(1-q^{2}\right) \ldots\left(1-q^{k}\right)}+\frac{\left(1-q^{n-1}\right)\left(1-q^{n-2}\right) \ldots\left(1-q^{n-k}\right)}{(1-q)\left(1-q^{2}\right) \ldots\left(1-q^{k-1}\right)} \\
& =\frac{\left(1-q^{n-1}\right)\left(1-q^{n-2}\right) \ldots\left(1-q^{n-k+1}\right)}{(1-q)\left(1-q^{2}\right) \ldots\left(1-q^{k-1}\right)}\left\{\frac{1-q^{n-k}}{1-q^{k}}+1\right\}
\end{aligned}
$$

which yields the desired result. (2.2) is a variation of the relatively well-known identities 


$$
q^{k}\left(\begin{array}{c}
n-1 \\
k
\end{array}\right)_{q}+\left(\begin{array}{l}
n-1 \\
k-1
\end{array}\right)_{q}=\left(\begin{array}{l}
n \\
k
\end{array}\right)_{q}
$$

and

$$
\left(\begin{array}{c}
n-1 \\
k
\end{array}\right)_{q}+q^{n-k}\left(\begin{array}{l}
n-1 \\
k-1
\end{array}\right)_{q}=\left(\begin{array}{l}
n \\
k
\end{array}\right)_{q}
$$

See, for instance Andrews [3].

\section{Connections with generalized Fibonacci numbers}

We finish with two results which extend the known formulas for these numbers, irrespective of their initial conditions, namely (3.1) and (3.2) below.

\section{Theorem 2.}

$$
\left(\frac{2-q^{k}-q^{n-k}}{1-q^{n}}\right) \alpha^{n} U_{n}=\alpha^{n-k} U_{n-k}+\alpha^{k} U_{k}
$$

Proof:

$$
\begin{gathered}
\left(\begin{array}{c}
n-1 \\
k
\end{array}\right)_{q}=\alpha^{k(n-k-1)} U_{n-k} \frac{U_{n-1} U_{n-2} \ldots U_{n-k+1}}{U_{1} U_{2} \ldots U_{k}} \\
=U_{n-k} C_{n, k} \alpha^{k(n-k-1)} \\
\left(\begin{array}{c}
n-1 \\
k-1
\end{array}\right)_{q}=\alpha^{\left(k-1 \_(n-k)\right.} U_{k} \frac{U_{n-1} U_{n-2} \ldots U_{n-k+1}}{U_{1} U_{2} \ldots U_{k}} \\
=U_{k} C_{n, k} \alpha^{(k-1)(n-k)}
\end{gathered}
$$

and the result follows after induction.

\section{Theorem 3.}

$$
\left(\begin{array}{l}
n \\
m
\end{array}\right)_{q}=U_{m+1} \alpha^{m}\left(\begin{array}{c}
n-1 \\
m
\end{array}\right)_{q}-q U_{n-m+1} \alpha^{n-m}\left(\begin{array}{c}
n-1 \\
m-1
\end{array}\right)_{q}
$$

Proof: It can readily be shown [15] that

$$
U_{n}=U_{m+1} U_{n-m}-q U_{m} U_{n-m-1}
$$

and so from Section 2

$$
U_{n} C_{n, k} \alpha^{m(n-m)}=U_{m+1}\left(U_{n-m} C_{n, k} \alpha^{m(n-m-1}\right) \alpha^{m}-q U_{n-m-1}\left(U_{m} C_{n, k} \alpha^{(m-1)(n-m}\right) \alpha^{n-m}
$$

and the required result comes from the use of the definition of $C_{n, k}$. This result is a generalization of equation $(F)$ in [14]. 


\section{Concluding comments}

There is a three-fold value in searching for elegant generalizations in number theory, namely, to investigate which identities are essential, to discover links with otherwise apparently unrelated results, and to formulate ideas for further research. Thus generalizing $q$-biomial coefficients to their multinomial analogues has provided the mathematical identities for scientific applications in seemingly unexpected contexts; for example, [2].

\section{References}

[1] Alexanderson, G. L. (1974). A Fibonacci analogue of Gaussian binomial coefficients. Fibonacci Quarterly. 12 (2), 129-132.

[2] Andrews, G. E., \& Baxter, R. J. (1987). Lattice gas generalization of the hard hexagon model. III. q-trinomial coefficients. Journal of Statistical Physics. 47 (3-4), 297-330.

[3] Andrews, G. E. (1970). A polynomial identity which implies the Rogers-Ramanujan identities. Scripta Mathematica. 28, 297-305.

[4] Andrews, G. E. (1974). Applications of basic hypergeometric functions. SIAM Review. 16 (4), 441-484.

[5] Carlitz, L. (1947). A problem of Dickson's. Duke Mathematical Journal. 14, 1139-1140.

[6] Carlitz, L. (1948). q-Bernoulli numbers and polynomials. Duke Mathematical Journal. 15, 987-1000.

[7] Carlitz, L. (1958). Expansions of $q$-Bernoulli numbers. Duke Mathematical Journal. 25, 355-364.

[8] Carlitz, L. (1961). Some integral equations satisfied by the complete integrals of first and second kind. Bolletino della Unione Matematica Italiana. (3) 16, 264-268.

[9] Carlitz, L. (1962). Generating functions for powers of certain sequences of numbers. Duke Mathematical Journal. 29, 521-537.

[10] Carlitz, L. (1968). A note on products of sequences. Bolletino della Unione Matematica Italiana. (4) 1, 362-365.

[11] Cross, J. T. (1983). The Euler $\varphi$-function in the Gaussian Integers. American Mathematical Monthly. 90 (8), 518-528.

[12] Gould, H. (1969). The bracket function and Fontene-Ward generalized binomial coefficients with application to Fibonomial coefficients. Fibonacci Quarterly. 7 (1), $23-40$.

[13] Hoggatt, V. E, Jr, \& Lind, D. A. (1968). Fibonacci and binomial properties of weighted compositions. Journal of Combinatorial Theory. 4, 121-124.

[14] Hoggatt, V. E. Jr. (1967). Fibonacci numbers and generalized binomial coefficients. The Fibonacci Quarterly. 5, 383-400. 
[15] Horadam, A. F. (1965). Basic properties of a certain generalized sequence of numbers. The Fibonacci Quarterly. 3, 161-176.

[16] Horadam, A. F. (1965). Generating functions for powers of a certain generalized sequence of numbers. Duke Mathematical Journal. 32, 437-446.

[17] Jerbic, S. K. (1968). Fibonomial Coefficients - A Few Summation Properties. San Jose State College, San Jose, California.

[18] Mercier, A. (1989). Identities containing Gaussian binomial coefficients. Discrete Mathematics. 76, 67-73. 